\title{
Genomic signatures and antiviral drug susceptibility profile of A(H1N1)pdm09
}

\author{
Marta Tiago Gíria ${ }^{a}$, Helena Rebelo de Andrade ${ }^{a, b}, *$, Luís André Santos ${ }^{b}$, Vanessa Martins Correia ${ }^{b}$, \\ Sónia Vicente Pedro ${ }^{c}$, Madalena Almeida Santos ${ }^{\mathrm{d}}$
}

a CPM-URIA, Faculdade de Farmácia da Universidade de Lisboa, Lisboa, Portugal

b Departamento de Doenças Infecciosas, Instituto Nacional de Saúde Dr Ricardo Jorge, Lisboa, Portugal

c Unidade de Tecnologia e Inovação, Departamento de Genética, Instituto Nacional de Saúde Dr Ricardo Jorge, Lisboa, Portugal

d Serviço de Patologia Clínica, Virologia/Biologia Molecular, Hospital de Curry Cabral, Lisboa, Portugal

\section{A R T I C L E I N F O}

\section{Article history:}

Received 8 July 2011

Received in revised form

20 September 2011

Accepted 8 November 2011

\section{Keywords:}

A(H1N1)pdm09

Viral fitness

Virulence

Internal proteins

Antiviral drug susceptibility

Risk assessment

\begin{abstract}
A B S T R A C T
Background: Genetic changes in influenza surface and internal genes can alter viral fitness and virulence. Mutation trend analysis and antiviral drug susceptibility profiling of $\mathrm{A}(\mathrm{H} 1 \mathrm{~N} 1)$ pdm09 viruses is essential for risk assessment of emergent strains and disease management.

Objective: To profile genomic signatures and antiviral drug resistance of $\mathrm{A}(\mathrm{H} 1 \mathrm{~N} 1) \mathrm{pdm} 09$ viruses and to discuss the potential role of mutated residues in human host adaptation and virulence.

Study design: A(H1N1)pdm09 viruses circulating in Portugal during pandemic and post-pandemic periods and 2009/2010 season. Viruses were isolated in MDCK-SIAT1 cell culture and subjected to mutation analysis of surface and internal proteins, and to antiviral drug susceptibility profiling.

Results: The A(H1N1)pdm09 strains circulating during the epidemic period in Portugal were resistant to amantadine. The majority of the strains were found to be susceptible to oseltamivir and zanamivir, with five outliers to neuraminidase inhibitors (NAIs) identified. Specific mutation patterns were detected within the functional domains of internal proteins PB2, PB1, PA, NP, NS1, M1 and NS2/NEP, which were common to all isolates and also some cluster-specific.

Discussion: Modification of viral genome transcription, replication and apoptosis kinetics, changes in antigenicity and antiviral drug susceptibility are known determinants of virulence. We report several point mutations with putative roles in viral fitness and virulence, and discuss their potential to result in more virulent phenotypes. Monitoring of specific mutations and genetic patterns in influenza viral genes is essential for risk assessing emergent strains, disease epidemiology and public health implications.
\end{abstract}

(c) 2011 Elsevier B.V. All rights reserved.

\section{Background}

The emergence of virulent influenza phenotypes is a consequence of genetic changes altering the function of individual proteins or their functional compatibility.

Genetic changes in the viral surface proteins can impact on virus binding, entry, assembly, release, induction of the host's immune response and antiviral drug resistance.

Within the internal proteins, genetic changes can impact on virulence and infectivity if they alter the ability of the virus to replicate or induce cellular apoptosis. ${ }^{1}$ The viral replication process is multigenic and interactive, involving the replication complex and the structural and functionally associated M1 and NS2/NEP proteins. ${ }^{1,2}$ The gene segments coding for the proteins involved in replication, code for additional proteins associated with the induction of

\footnotetext{
* Corresponding author. Tel.: +351217519332.

E-mail address: h.rebelo.andrade@insa.min-saude.pt (H. Rebelo de Andrade).
}

cellular apoptosis during viral infection. The (+1)ORF of PB1 encodes PB1-F2 with pro-apoptotic function in the host cells and the NS gene encodes NS1 protein associated with the activation of anti-apoptotic mechanisms, permitting viral replication to occur. ${ }^{3-5}$ The cassette of internal genes therefore controls the major processes by which viral fitness is determined: replication and apoptosis. An increased replication rate or stimulation of anti-apoptotic mechanisms, produces higher viral loads that can overcome host immune response, enhance infectivity and result in a more severe and transmissible disease. The most severe epidemiological situations caused by seasonal influenza viruses occurred in 1947 and 1951 as a consequence of genetic changes within internal proteins involved in replication of $A(H 1 N 1)$ viruses. The strains spread worldwide and caused exceptionally high mortality. ${ }^{6}$

Monitoring evolutionary trends in the genes encoding influenza virus internal proteins, and profiling antigenic proteins and antiviral drug susceptibility, are therefore essential to risk assessing influenza virulence, disease epidemiology and public health implications. 


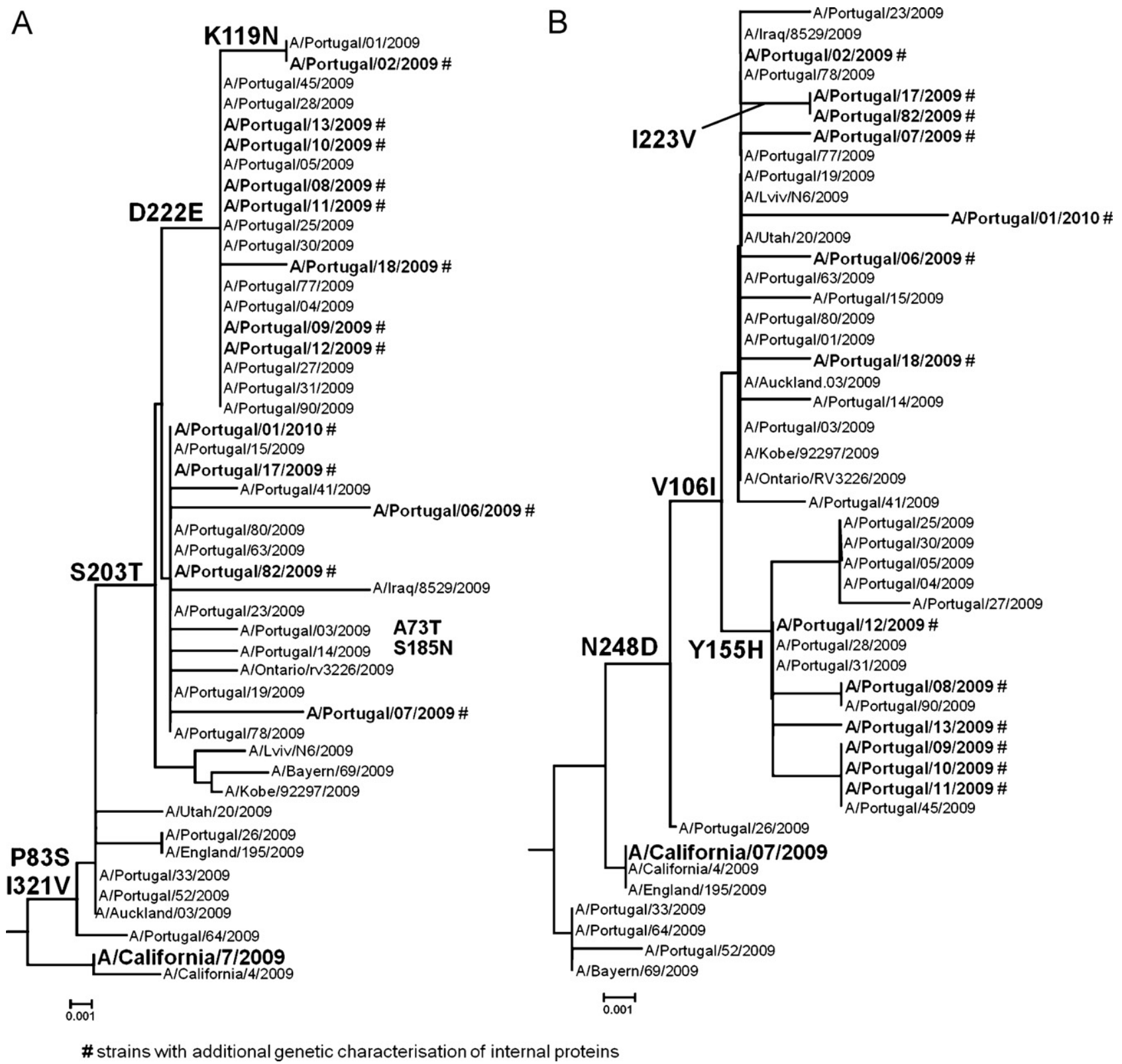

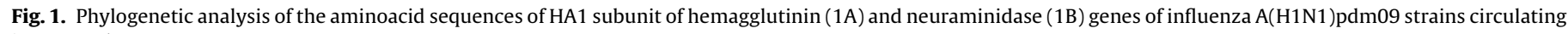
in Portugal.

\section{Objectives}

With the aim of risk assessing emergent strains, the main objectives of the study were to profile genomic signatures and antiviral drug susceptibility of A(H1N1)pdm09 viruses and discuss the potential putative role of mutated residues in human host adaptation and virulence.

\section{Study design}

A total of 110 specimens from laboratory-confirmed cases of pandemic influenza were collected in Portugal from July 2009 to January 2010. Strains were isolated in MDCK-SIAT1 cell culture. Antigenic surface glycoproteins and M1 and M2 proteins were genetically characterized and antiviral drug susceptibility was profiled. Genetic characterization of internal proteins was performed on viruses isolated from subsets of pregnant women, outliers to
NAIs and a 14 years old deceased patient with no risk factors. Cycle sequencing was performed based on adaptation of a protocol from CDC, recommended by WHO. ${ }^{7}$ Genetic mutation analysis and phylogenetic analysis were performed with LasergeneV.4.05DNASTAR and MEGA4.0, with comparison to sequences of the A(H1N1)pdm09 2010/2011 vaccine strain (A/California/7/2009) and published reference $A(H 1 N 1)$ pdm09 strains circulating worldwide within pandemic and post-pandemic periods. Phenotypic evaluation of antiviral drug susceptibility was performed by fluorescent assay with MUNANA substrate as previously described. ${ }^{8}$ Minor and major phenotypic outliers were identified through the establishment of lower and upper $\mathrm{IC}_{50}$ cut offs values. ${ }^{8}$ Genotypic evaluation of antiviral drug susceptibility to NAIs and to amantadine was performed by analysing NA and M2 protein sequences, respectively. In the M2 sequence the 5 well-defined molecular markers of resistance to amantadine were analysed: L26F/I; V27A/D; A30T; S31N and G34E. 


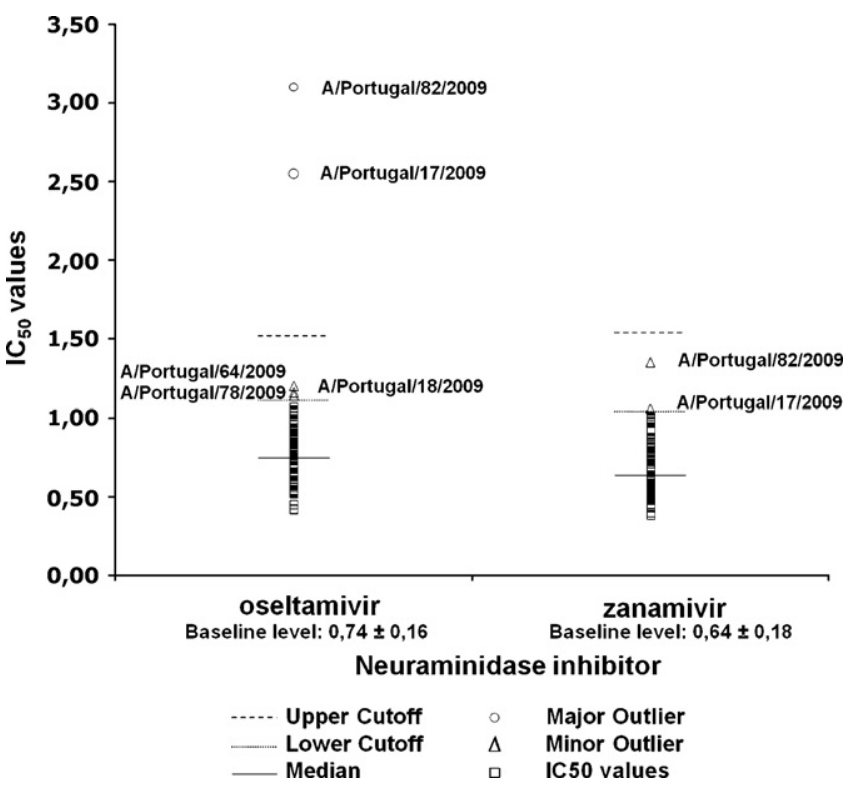

Fig. 2. Phenotypic evaluation of A(H1N1)pdm09 virus susceptibility to NAIs by fluorescence assay.

\section{Results}

Virus isolation was performed for all $110 \mathrm{~A}(\mathrm{H} 1 \mathrm{~N} 1)$ pdm09 cases. Of these, 37 were genetically characterized for antigenic glycoproteins and for $\mathrm{M}$ protein. Antiviral drug susceptibility profile was phenotypically evaluated for 103 of the 110 isolates. Ninetyeight were further sequenced for genotypic evaluation of NAIs susceptibility and 96 for amantadine susceptibility. Genetic characterization of internal proteins PB2, PB1, PB1-F2, PA, NP, NS1 and NS2/NEP was performed for 13 of the 37 isolates, including viruses isolated from cases in the subsets described above.

Within the HA1 domain of the HA, all 37 strains were shown to contain the mutations P83S and I321V (Fig. 1A). Thirty-three of the 37 strains had the substitution S203T, and 19 also contained D222E. Additional point mutations were observed, including A73T and $\mathrm{S} 185 \mathrm{~N}$ in individual strains, which are located in the putative antigenic sites $\mathrm{Cb}$ and $\mathrm{Sb}$, respectively. The substitution K119N which results in the creation of an $\mathrm{N}-\mathrm{X}-\mathrm{S}$ motif, was observed in 2 strains. In the NA gene, 34 of the 37 strains analysed contained N248D and V106I substitutions (Fig. 1B). Fifteen strains had Y155H and two contained I223V in the NA gene.

The genetic characterization of MP of 37 strains identified M128L, E201D and M203I substitutions in individual isolates compared to the A(H1N1)pdm09 2010/2011 vaccine strain MP sequence, located within the M1 coding region.

Phenotypic evaluation of NAIs susceptibility revealed three minor and two major outliers to oseltamivir, as shown in Fig. 2. The three minor outliers exhibited reduced susceptibility of approximately two-fold and the two major outliers of approximately 3and 4-fold, compared to baseline level. The two major outliers were also found to be minor outliers to zanamivir (MjOOsel/MnOZana), with reduced susceptibility of approximately two-fold compared to baseline level. In the NA sequence of these two MjOOselt/MnOZana strains, the mutation I223V was observed. Genotypic evaluation of susceptibility to amantadine revealed that all 96 strains analysed had an aspargine $(\mathrm{N})$ at position 31 in the M2 coding region.

The genetic profile of the internal proteins of $13 \mathrm{~A}(\mathrm{H} 1 \mathrm{~N} 1) \mathrm{pdm} 09$ isolates analysed revealed substitutions P224S in PA, V100I and L122Q in NP and I123V in NS1, which were common to all 13 isolates (highlighted in bold and \#, Fig. 1), as shown in Table 1. PB1-F2 was present in the truncated non-functional form of 11 amino acids.

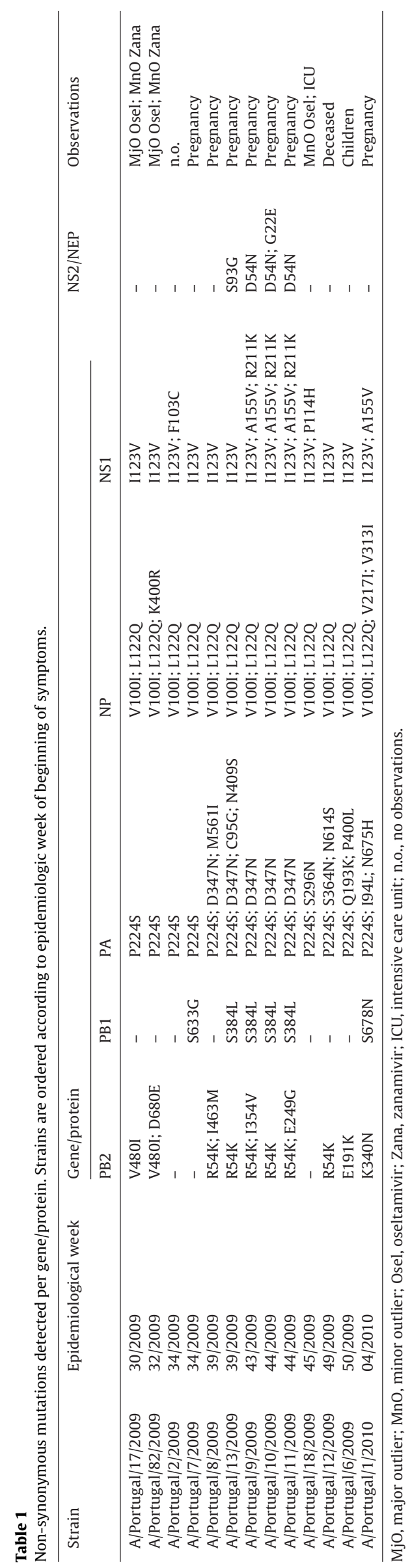


Viruses isolated from the subset of pregnant women, were observed to have R54K substitution in PB2 in 6 cases, 5 of which also contained D347N in PA. Of these, 4 also contained S384L in PB1 and a small cluster of 3 isolates was characterized by the additional substitutions A155V and R211K in NS1, and D54N in NS2/NEP.

The mutation V480I was found only in the PB2 sequence of the two strains exhibiting a reduced susceptibility profile to both NAIs (MjOOselt/MnOZana).

\section{Discussion}

A(H1N1)pdm09 viruses characterized by the HA substitution S203T and both N248D and V106I in NA, were found to be the dominant circulating strain throughout the epidemic period (2009-2010) in Portugal. Previous studies have also reported dominant circulation of this strain worldwide and suggested this is a consequence of enhanced viral fitness. ${ }^{9,10}$

Within HA1, there was no evidence of evolutionary trends, in agreement with the genetic and antigenic homogeneity of $\mathrm{A}(\mathrm{H} 1 \mathrm{~N} 1)$ pdm09. ${ }^{11}$ The location of amino acid substitutions observed in HA1 however, suggests these could result in phenotypic changes; the D222E substitution is located within a loop of the receptor-binding site, A73T and S185N within the putative antigenic sites $\mathrm{Cb}$ and $\mathrm{Sb}$, respectively, and $\mathrm{K} 119 \mathrm{~N}$ has been reported to result in creation of a potential $\mathrm{N}$-glycosylation site. ${ }^{9,12}$ Correlation of these mutations with particular phenotypes, binding specificity or clinical outcome of infection however, needs further investigation.

In the internal genes, the V100I and L122Q substitutions observed in NP, I123V in NS1 and P224S in PA characterize the genetic profile of all isolates analysed, reflecting the dominant circulation of this $\mathrm{A}(\mathrm{H} 1 \mathrm{~N} 1)$ pdm09 genetic variant in Portugal within the period analysed. Residues 100 and 122 in NP are located within the body domain of the protein and are thought to be involved in PB2 and PB1-NP interaction, which is crucial for RNA replication. The amino acid substitution V100I was associated with the raising of the pandemic alert from phase 4 to 6 , and is a human to avian signature change known to translate into enhanced viral fitness probably by increasing viral transmissibility or infectivity. ${ }^{10,13}$ I123V in NS1 is located in the effector domain, a functional region regulating cellular apoptosis, and therefore suspected to play a role in adaptation to the human host and to increase virulence..$^{10} \mathrm{P} 224 \mathrm{~S}$ in PA is located in the N-terminal domain, which has endonuclease activity. Although no phenotypic outcome for this mutation has been established to date, endonuclease activity is critical for initiating transcription and therefore, genetic changes in this region might be expected to alter replication kinetics. ${ }^{14}$ Although the phenotypic outcome of L122Q has not been established, this mutation has been previously reported as a virulence factor in H5N1 by greatly increasing replication. ${ }^{15}$

In the viruses from pregnant women, in addition to the mutations common to all isolates analysed, a unique profile of 6 genetic signatures in the functional domains of internal proteins defined a specific cluster. Considering their genetic location, they present a high potential for translating into phenotypic changes in fitness and virulence. Both R54K in PB2 and D347N in PA are located within functional domains for binding PB1.,18 Since the function of the polymerase proteins depends on the formation of a heterodimer for optimal viral RNA replication and transcription, these substitutions together with P224S in PA, may have impacted on polymerase subunit binding to PB1 and have had an unpredictable effect on replication in these strains. ${ }^{16,17}$ Within NS1, the cluster is defined by $\mathrm{A} 155 \mathrm{~V}$ and $\mathrm{R} 211 \mathrm{~K}$ substitutions, both located within the C-terminal of the effector domain, as is I123V, which has previously been found to regulate cellular apoptosis. ${ }^{5,18}$ Replication and apoptosis are the major mechanisms that define viral fitness. The occurrence of different point mutations as occurred in strains in this cluster, could be compensatory or have had a cumulative effect leading to a different phenotype. These mutations will continue to be monitored in non-pregnant patients to evaluate whether they are subset exclusive, resulting from enhanced function of individual proteins or increasing functional compatibility of proteins.

The virus isolate from the deceased patient contained a distinctive set of mutations which could have contributed to this more severe clinical outcome. In addition to N248D and V106I in NA, S203T in HA, V100I in NP and I123V in NS1, this isolate contained L122Q in NP, S364N and N614S in PA, and R54K in PB2, all located in PB1 binding domains of the respective proteins. ${ }^{17}$ A direct association of PB1 binding to the other polymerase subunits and to $\mathrm{NP}$ with a more virulent phenotype has not been established to date. However, the formation of PB2-PB1-PA heterodimer and the binding of PB2 and PB1-NP into ribonucleoproteins both determine vRNA transcription and cRNA synthesis for genome replication and therefore, have the potential to greatly affect the viral replication cycle. $^{17}$

Within the subset of outliers to NAIs, the two MjOOsel/MnOZana were defined by I223V in NA and V480I in PB2. Amino acid substitutions at position 223 in NA have previously been shown to affect virus susceptibility to both oseltamivir and zanamivir, in recent $\mathrm{A}(\mathrm{H} 1 \mathrm{~N} 1)$ pdm09 and seasonal viruses. ${ }^{19,20}$ Residue 223 is located in the framework of the NA active site, and thus interacts with the catalytic residues to which antiviral drugs bind. ${ }^{21}$ The contribution of V480I in PB2 to the reduced susceptibility profile observed is unclear. In addition, no phenotypic impact was observed in $\mathrm{A}(\mathrm{H} 1 \mathrm{~N} 1)$ pdm09 viruses containing NA mutation Y155H, previously observed in seasonal $\mathrm{A}(\mathrm{H} 1 \mathrm{~N} 1)$ viruses resistant to both NAIs. $^{22}$

Correlating specific mutations with virulent phenotypes in the human host is not straightforward. Animal models differ in their immune response to infection and multiple other factors interfere with clinical and epidemiology outcomes of infection. Also, the genetic background of specific mutations is divergent among influenza strains and can have different phenotypic outcomes. Several mutations have however, been recognized as virulence markers. Although some mechanisms remain unclear, the modification of genome transcription, replication, apoptosis and antigenicity are determinants of virulence. Additionally to known molecular markers of virulence, we have placed selected residues within functional domains of internal proteins that may enhance viral fitness or increase virulence under genomic surveillance.

\section{Funding}

Calouste Gulbenkian Foundation (Fundação Calouste Gulbenkian) research grant funded the experimental work and the Portuguese Science and Technology Foundation the doctoral grants (SFRH/BD/65211/2009; SFRH/BD/62676/2009; SFRH/BD/48532/2008).

\section{Competing interest}

None declared.

\section{Ethical approval}

No human subjects were used in this research study. Specimens were accessed through anonymous banks, reviewed and exempt for specific issue document from the Ethic Commission of the National Institute of Health, Lisbon, Portugal. 


\section{Acknowledgments}

The authors would like to thank Dr. Joanna Ellis from the Health Protection Agency in London for the revision of the manuscript.

\section{References}

1. Basler C, Aguilar P. Progress in identifying virulence determinants of the 1918 H1N1 and the Southeast Asian H5N1 influenza A viruses. Antiviral Res 2008;79:166-78.

2. Chen L, Davis TC, Zhou H, Cox NJ, Donis RO. Genetic compatibility and virulence of reassortants derived from contemporary avian H5N1 and human H3N2 influenza A viruses. PLoS Pathogens 2008;4(5):e1000072.

3. Hai R, Schmolke M, Varga Z, Manicassamy B, Wang TT, Belser JA, et al. PB1-F2 expression by the 2009 pandemic H1N1 influenza virus has minimal impact on virulence in animal models. J Virol 2010;84(9):4442-50.

4. Hale BG, Randall RE, Ortín J, Jackson D. The multifunctional NS1 protein of influenza A viruses. J Gen Virol 2008;89(Pt 10):2359-76.

5. Zhirnov OP, Konakova TE, Wolff T, Klenk HD. NS1 protein of influenza A virus down-regulates apoptosis. J Virol 2002;76(4):1617-25.

6. Nelson MI, Viboud C, Simonsen L, Bennett RT, Griesemer SB, St George K, et al. Multiple reassortment events in the evolutionary history of H1N1 influenza A virus since 1918. PLoS Pathogens 2008;4(2):e1000012.

7. World Health Organization. Sequencing primers and protocol; 2009. Available at: http://www.who.int/csr/resources/publications/swineflu/sequencing_primers/ en/index.html (accessed 15.10.10)

8. Correia V, de Andrade HR, Santos LA, Lackenby A, Zambon M. Antiviral drug profile of seasonal influenza viruses circulating in Portugal from 2004/2005 to 2008/2009 winter seasons. Antiviral Res 2010;86:128-36.

9. Igarashi M, Ito K, Yoshida R, Tomabechi D, Kida H, Takada A. Predicting the antigenic structure of the pandemic (H1N1) 2009 influenza virus hemagglutinin. PLoS One 2010;5(1):e8553.

10. Pan C, Cheung B, Tan S, Li C, Li L, Liu S, et al. Genomic signature and mutation trend analysis of pandemic (H1N1) 2009 influenza A virus. PLoS One 2010;5(3):e9549.
11. World Health Organization. Recommended viruses for influenza vaccines for use in the 2011 southern hemisphere influenza season; 2010. Available at http://www.who.int/csr/disease/influenza/201009_Recommendation.pdf (accessed 16.12.10).

12. Kilander A, Rykkvin R, Dudman SG, Hungnes O. Observed association between the HA1 mutation D222G in the 2009 pandemic influenza $A(H 1 N 1)$ virus and severe clinical outcome, Norway 2009-2010. EuroSurveill 2010;15(9), pii $=19498$.

13. Wu R, Zhang H, Yang K, Liang W, Xiong Z, Liu Z, et al. Multiple amino acid substitutions are involved in the adaptation of H9N2 avian influenza virus to mice. Vet Microbiol 2009;138:85-91.

14. Liu Y, Lou Z, Bartlam M, Rao Z. Structure-function studies of the influenza virus RNA polymerase PA subunit. Sci China Ser C Life Sci 2009;52(5):450-8.

15. Tada T, Suzuki K, Sakurai Y, Kubo M, Okada H, Itoh T, et al. NP Body Domain and PB2 Contribute to Increased Virulence of H5N1 Highly Pathogenic Avian Influenza Viruses in Chickens. J Virol 2011;85(4):1834-46.

16. Guu TS, Dong L, Wittung-Stafshede P, Tao YJ. Mapping the domain structure of the influenza $A$ virus polymerase acidic protein (PA) and its interaction with the basic protein 1 (PB1) subunit. Virology 2008;379:135-42.

17. Perez DR, Donis RO. Functional analysis of PA binding by influenza A virus PB1: effects on polymerase activity and viral infectivity. J Virol 2001;75(17):8127-36.

18. Salahuddin P, Khan AU. Structural and functional analysis of NS1 and NS2 Proteins of H1N1 subtype. Gen Prot Bioinform 2010;8(3):190-9.

19. Hayden FG, de Jong MD. Emerging influenza antiviral resistance threats. J Infect Dis 2011;203(1):6-10.

20. Deyde VM, Sheu TG, Trujillo AA, Okomo-Adhiambo M, Garten R, Klimov AI, et al. Detection of molecular markers of drug resistance in 2009 pandemic influenza A (H1N1) viruses by pyrosequencing. Antimicrob Agents Chemother 2010;54(3):1102-10.

21. Yen HL, Hoffmann E, Taylor G, Scholtissek C, Monto AS, Webster RG, et al. Importance of neuraminidase active-site residues to the neuraminidase inhibitor resistance of influenza viruses. J Virol 2006;80(17):8787-95.

22. Monto AS, McKimm-Breschkin JL, Macken C, Hampson AW, Hay A, Klimov A, et al. Detection of influenza viruses resistant to neuraminidase inhibitors in global surveillance during the first 3 years of their use. Antimicrob Agents Chemother 2006;50(7):2395-402. 